Chirurg 2012 $\cdot 83: 482$

DOI 10.1007/s00104-012-2309-7

Online publiziert: 11 . Mai 2012

๑) Springer-Verlag 2012

T. Franzke $\cdot J$. Jähne

Klinik für Allgemein- und Viszeralchirurgie, Schwerpunkt für endokrine und onkologische Chirurgie,

Diakoniekrankenhaus Henriettenstiftung, Hannover

\title{
Überlebensvorteil von Gastrektomie mit Metastasenresektion
}

\author{
Bei Patienten mit metastasiertem \\ Magenkarzinom unter Chemotherapie
}

Tumorreduktion oder zur Symptompalliation durchgeführt (R1/2-Resektion). Gruppe C $(n=185)$ bestand aus Patienten, die lediglich chemotherapiert wurden (ggf. mit Bypassoperation).

\section{Ergebnisse}

\section{Hintergrund}

Trotz abnehmender Inzidenz und Mortalität bleibt das Magenkarzinom (GC) die zweithäufigste Ursache von Krebstodesfällen weltweit. Die Standardbehandlung von Patienten mit Magenkarzinomen und Fernmetastasen ist weiterhin die palliative Chemotherapie sowie „best supportive care" mit jedoch schlechten einjährigen Überlebensraten. Daher untersucht die vorliegende Arbeit den erzielten Benefit von Gastrektomie mit und ohne Metastasenentfernung in Kombination mit palliativer Chemotherapie.

\section{Methodik}

Retrospektiv wurden 2 Datenbanken ausgewertet. 2283 Patienten mit Magenkarzinomen wurden zwischen 2003 und 2008 identifiziert. 274 Patienten mit StadiumIV-Karzinomen und durchgeführter Chemotherapie wurden in die Studie aufgenommen. In Gruppe A $(\mathrm{n}=42)$ wurden der Primarius sowie die Metastasen operativ entfernt (R0-Resektion). In Gruppe $B(n=47)$ wurde eine palliative Gastrektomie („debulking“) zum Zwecke der
Eine lokale Peritonealkarzinose war die häufigste Metastasierung (63\%), gefolgt von intraabdominellen Lymphknotenmetastasen (44\%) und Lebermetastasen (26\%). In 19\% waren 2 Organssysteme betroffen.

Das mediane Überleben aller Patienten betrug 11,8 Monate (Gruppe A: 28 Monate; Gruppe B: 15,5 Monate; Gruppe C: 9 Monate). In Gruppe A waren 12 (29\%) Patienten in einem mediane Follow-up von 29,1 Monaten weiterhin tumorfrei.

\section{Diskussion und Fazit}

Der Stellenwert der Chirurgie bei Patienten mit metastasiertem Magenkarzinom wird weiterhin kontrovers diskutiert. Die aktuellen Leitlinien raten lediglich bei symptomatischen Patienten zu einem chirurgischen Vorgehen.

Die vorliegende retrospektive Studie zeigt, dass die chirurgische Therapie (Gastrektomie und Metastasenresektion) in Kombination mit einer systemischen Chemotherapie einen signifikanten Überlebensvorteil für Patienten mit metastasiertem Magenkarzinom erzielen kann. Von immenser Wichtigkeit hinsichtlich des tumorfreien Überlebens scheint weiterhin die RO-Resektion. Die starke Selektierung des Patientenguts, das uneinheitliche chirurgische Vorgehen, die Vereinheitlichung der unterschiedlichen Metastasierungen sowie uneinheitliche Chemotherapieregime lassen jedoch Zweifel an der Validität der Ergebnisse aufkommen. Auch die Autoren sehen erheblich methodische Schwächen in ihrer Studien und fordern die Durchführung weiterer prospektiver Studien.

\section{Korrespondenzadresse}

\section{Dr. T. Franzke}

Klinik für Allgemein- und Viszeralchirurgie, Schwerpunkt für endokrine und onkologische Chirurgie, Diakoniekrankenhaus Henriettenstiftung, Marienstr. 72-90, 30171 Hannover Timm.Franzke@ddh-gruppe.de

Interessenkonflikt. Der korrespondierende Autor gibt für sich und seinen Koautor an, dass kein Interessenkonflikt besteht. 